\title{
The Evolution of the Mitochondria-to-Calcium Release Units Relationship in Vertebrate Skeletal Muscles
}

\author{
Clara Franzini-Armstrong ${ }^{1}$ and Simona Boncompagni ${ }^{2}$ \\ ${ }^{1}$ Department of Cell and Developmental Biology, School of Medicine, University of Pennsylvania, Philadelphia, PA 19104, USA \\ ${ }^{2}$ Center for Research on Ageing and Department of Neuroscience and Imaging, Università Gabriele d'Annunzio, \\ 66100 Chieti, Italy
}

Correspondence should be addressed to Clara Franzini-Armstrong, armstroc@mail.med.upenn.edu

Received 5 July 2011; Accepted 10 August 2011

Academic Editor: Aikaterini Kontrogianni-Konstantopoulos

Copyright (C 2011 C. Franzini-Armstrong and S. Boncompagni. This is an open access article distributed under the Creative Commons Attribution License, which permits unrestricted use, distribution, and reproduction in any medium, provided the original work is properly cited.

The spatial relationship between mitochondria and the membrane systems, more specifically the calcium release units (CRUs) of skeletal muscle, is of profound functional significance. CRUs are the sites at which $\mathrm{Ca}^{2+}$ is released from the sarcoplasmic reticulum during muscle activation. Close mitochondrion-CRU proximity allows the organelles to take up $\mathrm{Ca}^{2+}$ and thus stimulate aerobic metabolism. Skeletal muscles of most mammals display an extensive, developmentally regulated, close mitochondrionCRU association, fostered by tethering links between the organelles. A comparative look at the vertebrate subphylum however shows that this specific association is only present in the higher vertebrates (mammals). Muscles in all other vertebrates, even if capable of fast activity, rely on a less precise and more limited mitochondrion-CRU proximity, despite some tethering connections. This is most evident in fish muscles. Clustering of free subsarcolemmal mitochondria in proximity of capillaries is also more frequently achieved in mammalian than in other vertebrates.

\section{Introduction}

Mitochondria have two functional requirements: they need oxygen and also some stimulation by $\mathrm{Ca}^{2+}[1-3]$. The latter event has been disputed for a while, but it is now clear that mitochondria take up some of the $\mathrm{Ca}^{2+}$ released from the endoplasmic reticulum (ER) under physiological conditions, a step that has important effects on a variety of their functions, including the stimulation of aerobic metabolism. The uptake has been initially difficult to pinpoint, but specifically targeted aequorin [4] gave a first clear evidence for $\mathrm{Ca}^{2+}$ entry into the mitochondria of living cells, and fluorometric measurements of mitochondrial dehydrogenases activity provided an early fast measurement of mitochondrial $\mathrm{Ca}^{2+}$ uptake in vivo [5]. The concept has been developed that this uptake is strictly dependent on a planned proximity between the organelles and certain components of the ER. The socalled high $\mathrm{Ca}^{2+}$ microdomain hypothesis proposes that the proximity is necessary because the relatively low mitochondrial affinity for $\mathrm{Ca}^{2+}[1,6-8]$ means that uptake will occur only if the mitochondrion is near the source, so that it experiences a sudden increase of the cytoplasmic $\mathrm{Ca}^{2+}$ level to relatively high values [9-11]. Close proximities between ER and mitochondria are indeed frequent and well documented in liver cells, where the ER, both RER and SER, is frequently tightly wrapped around the mitochondria profiles $[12,13]$ even in organisms as low as fish. Visible tethering connections between ER and mitochondria are sufficiently strong in liver and myocardium of mammals to maintain the association during cellular fractionation [14, 15]. More recently, an even more direct interaction between ER components and the outer mitochondrial membrane has been proposed, based on a physical link between the voltage-dependent anion channel (VDAC) of the outer mitochondrial membrane and Ip3 receptors of the ER, allowing direct coupling between $\mathrm{Ca}^{2+}$ release and mitochondrial uptake [16].

Cardiac and skeletal muscle cells are inherently crowded due to their content of myofibrils, and the mitochondria share the narrow intermyofibrillar spaces with the sarcoplasmic reticulum (SR); transverse (T) tubules; dyads/triads; 
glycogen granules and lipid droplets as well as the basic cytoskeletal network. Among these organelles, the triads/dyads, constituted of junctional SR (jSR) and T tubules, function as calcium release units (CRUs) through which $\mathrm{Ca}^{2+}$ exits the SR during muscle activation. CRUs are located at specific and frequent intervals and form a discontinuous ring around the myofibrils following the path of the T tubule network. Given the narrowness of the shared spaces, it is not surprising that mitochondria and CRUs often rub elbows. In skeletal and cardiac muscles of rodents, the proximity is sufficient for mitochondria to take up $\mathrm{Ca}^{2+}$ even during the time course of a single twitch $[17,18]$. Even though the uptake by individual organelles is small, it can affect the rate of relaxation in fibers that are rich in mitochondria [19] and in rat fibers the buffering action of mitochondria may be sufficient to reduce the frequency of detectable spontaneous releases [20].

Pertinent questions are (1) is the CRU-mitochondria relationship random, or is there a higher structural hierarchy that specifically establishes the relative positioning of the two organelles in skeletal muscle and (2) is the same structural relationship present in muscles of all vertebrates. Structural and functional evidence points to a planned 3-D relationship between the two organelles $[21,22]$ but not for all mitochondria and perhaps not in all muscles. Taking advantage of different kinetic properties of $\mathrm{Ca}^{2+}$ chelators, Shkryl and Shirokova [23] demonstrated that two functional categories of mitochondria coexist in skeletal muscle of the rat: those that take up some of the $\mathrm{Ca}^{2+}$ released by the SR during muscle activation even in the presence of fast $\mathrm{Ca}^{2+}$ chelators and those that do not. The former are, it must be presumed, closely juxtaposed to CRUs, the latter are at some distance. The structural equivalent of this functional phenomenon is the well-specified position of mitochondria relative to CRUs in the muscle fibers from the same species, as well as from other mammals. Muscles of course are of primary importance in any activity from food gathering, to eating, to defense and, indirectly, to reproduction and in some cases to heat production. Based on the fundamental tenet of evolution that features offering survival advantage are retained, it may be expected that any variation essential for the effective performance of muscles would appear as an early event in evolution. As an example, the orderly arrangement of thin and thick filaments in cross-striated sarcomeres, offering the possibility of rapid movements, is an early evolutionary event, that is found in insects, all chordates, and, even, in a slightly rudimentary form in some molluscan muscles. An early appearance of a tight mitochondrion/CRU relationship in vertebrate evolution would indicate that this particular arrangement is of overriding functional usefulness to both mitochondria and skeletal muscle; a later appearance would indicate that it is advantageous but not essential.

In order to establish the evolutionary significance of specific mitochondria/CRU relationships, we launched a widespread exploration of muscle ultrastructure within the vertebrate subphylum. To that effect, we examined muscle samples from a few organisms in each class, from fish to mammals, relying on the existing literature for further data.

\section{Materials and Methods}

Skeletal muscles were fixed in a variety of vertebrates. Most images came from an extensive archive present in the laboratory, other from muscles that were fixed specifically for this project. Fish: Lampetra planeri (larvae); Eptatretus stouti (Hagfish); Lepisosteus osseus (Gar fish); Citharichthys sordidus (pacific sand dab); Danio rerio (zebrafish); Poecilia reticulata (guppy); Poecilia latipinna var. (black molly), toadfish (Opsanus tau); amphibia (Rana pipiens, R. temporaria); Reptiles (Boa constrictor, Nerodia sipedon, Anolis carolinensis); Birds (Meleagris gallopavo; fringilla sp., Gallus gallus); Mammals (Mus rattus, Rattus sp., Felis catus).

The animals were euthanized by a variety of means (cervical dislocation and/or an overdose of anesthetic: $\mathrm{CO}_{2}$, ether, isoflurane, sodium pentobarbital). After euthanasia, the muscles were exposed and either fixed in situ by dripping the fixative on them, or carefully dissected tendon-totendon, pinned in Sylgard dish (Dow Corning) at resting length and immersed in fixative. Fixation was in 3-9\% glutaraldehyde in $0.1 \mathrm{M}$ cacodylate buffer ( $\mathrm{pH}$ 7.2) at room temperature. The muscles were stored in fixative at $4{ }^{\circ} \mathrm{C}$ for variable periods of time, then postfixed in $2 \% \mathrm{OsO}_{4}$ in the same buffer for $1-2 \mathrm{hr}$ at $4^{\circ} \mathrm{C}$, en block stained in saturated uranyl acetate, with several washes after each step, and embedded in Epon 812. Muscles for domestic chicken and kangaroo (Macropus sp.) were simply obtained from the supermarket, and small samples were treated as the freshly dissected muscles. Ultrathin sections (about $40 \mathrm{~nm}$ ) were cut in an ultramicrotome Leica Ultracut R (Leica Microsystem, Austria) using a Diatome diamond knife (Diatome Ltd. Biel, Switzerland) and stained in uranyl acetate and lead citrate solutions.

\section{Results and Discussion}

3.1. Mammals. The following description, based on published electron micrographs of various muscles from laboratory rat and mouse $[22,24-26]$ and extensive unpublished observations by the two authors, offers the background for the overall distribution of mitochondria in muscles of placental mammals. Many mitochondria in these muscles lie within transverse planes that are positioned in close proximity of the triads (CRUs), between them and the nearby $\mathrm{Z}$ line, and thus have a preferential location opposite to the I bands of the sarcomere (Figure 1(a)). These mitochondria are very thin and elongated and closely follow the junctional SR sacs of CRUs over long distances, frequently coming to distances of $\sim 25 \mathrm{~nm}$ from the SR surface (Figure 1(b); [22]. Some fibers (e.g., type IIX and IIB in mouse) have almost exclusively this type of mitochondrial disposition. A second additional set of mitochondria is present in fibers that are richer in these organelles (e.g., type I and IIA in mouse). These mitochondria are larger in diameter; they are longitudinally oriented and may span the distance of several sarcomeres, encompassing $\mathrm{I}$ and $\mathrm{A}$ bands and $\mathrm{Z}$ lines. Appropriate sections show that these mitochondria extend transversely oriented arm-like branches as they pass at the level of the I band and these branches are those that follow along the length of triads (Figure 1(b)). It is not clear 


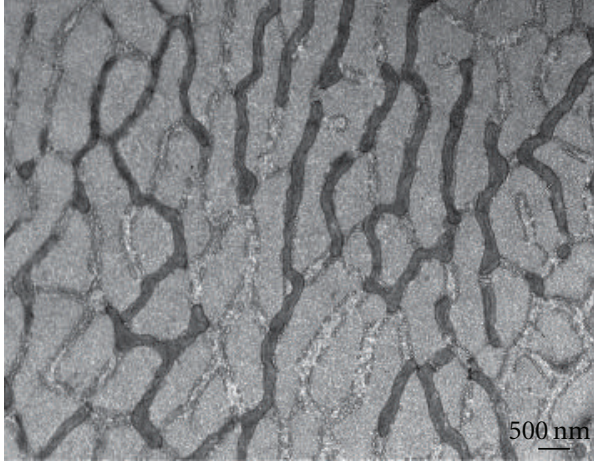

(a)

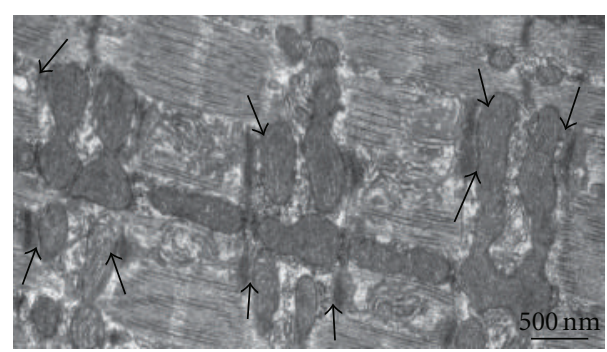

(b)

Figure 1: Cross (a) and longitudinal (b) sections of muscle fibers from the mouse EDL muscle. In (a), slender, elongated approximately cylindrical mitochondria profiles occupy the intermyofibrillar spaces, running transversely for long distances between the myofibrils at the I band level. In (b), the longitudinal axis of the fiber runs from left to right at a slight angle. Longitudinally arranged mitochondria send out transverse branches at the level of the I band, that correspond to those seen in (a). The transverse mitochondria branches run parallel to triads (CRUs) and are closely opposed to the junctional SR (arrows) over long distances.

whether all the longitudinally arranged mitochondria are directly connected to transverse extensions, but in general it can be assumed that these two sets of mitochondria are part of the same continuum. A different set of mitochondria (not shown) resides at peripheral sites, between the most peripheral myofibrils and the plasmalemma. These mitochondria are piled up into irregular mounds that project over the fiber surface and they are closely apposed to each other, but at some distance from the nearest myofibrils and CRUs. Capillaries are usually located in proximity of these peripheral clusters of mitochondria, so that these organelles are not near sites of $\mathrm{Ca}^{2+}$ release from the SR, but they are in close proximity to the capillaries. Indeed, peripheral grouping of mitochondria in mammalian fibers is mostly present in "red," richly vascularized muscles. These peripheral mitochondria may not "sense" the SR $\mathrm{Ca}^{2+}$ release, but are near oxygen sources. Unfortunately, although the mitochondria content of more exotic mammal, such as the cheetah known for the fastest running speed, has been explored [27], no information of the actual positioning of mitochondria relative to CRU is available for these muscles.

Two pieces of evidence indicate that the specific targeting of mitochondria at the I band and their structural coupling to CRUs in mammalian muscles are not due to chance.
First is the fact that association of mitochondria with CRUs is acquired as a developmentally regulated event during postnatal differentiation in mouse [22] and during a recapitulation of these events in the recovery of human denervated atrophic muscle induced by electrical stimulation [28]. Secondly, strong connecting tethers link the junctional SR of CRUs to adjacent mitochondria (Figures 2(a) and 2(c), arrows) $[22,26]$. The tethers link directly the SR membrane to the outer mitochondrial membrane and seem to be strong enough to hold them together if they are pulled apart (such as if the fiber is exposed to hypotonic solutions).

An extensive mitochondrion-CRU association has been detected in muscles from the domestic cat (unpublished observations): from the guinea pig [29] and from human muscles $[21,30,31]$. Taken together, these facts indicated that a major subset of mitochondria in mammalian muscle are in a specifically planned close structural and functional proximity to the SR, and more specifically with the domains (CRUs) that are responsible for $\mathrm{Ca}^{2+}$ release during excitation-contraction coupling. The association of the selected mitochondria with CRUs is very extensive, since approximately a quarter of the elongated mitochondrion outer surface is in very close proximity to a jSR element and is maintained by connecting tethers. This disposition seems to be common to muscles from placental mammalian that have been examined by ultrastructure.

It can also be inferred that the mitochondria that are not tethered to CRUs may be free to move and perhaps the peripatetic organelles are those aggregated in the subplasmalemmal spaces. Their presence is driven to the proximity of capillaries by the necessity of a high anaerobic profile. Indeed, the highly aerobic diaphragm of the smallest mammal, the shrew, is intensively vascularized and displays an extensive array of peripheral mitochondria [32].

One notable exception to the specific mitochondrionCRU alignment in mammalian muscles is found in the superfast cricothyroid muscle that produces the ultrasound used by the bats for echolocation [33]. In these muscles, the mitochondria are not at the triads, but are located in long longitudinal lines between the myofibrils, a disposition that is typical of all nonmammalian vertebrates (see below).

Additionally, a muscle from a marsupial also shows no specific relationship between mitochondria positioning and CRUs (unpublished observations).

3.2. Birds. Birds, like mammals, are homoeothermic, and some of them exhibit very fast and continuously active muscles. It would be expected that if specific relationships between mitochondria and CRUs and between mitochondria and capillaries were of overriding functional importance, such relationships would be found in muscles from this group. We were surprised to find that pectoral muscles in domestic chicken and turkey (admittedly not very active muscles), and also the leg and flight muscles of finches (that are quite active) display mitochondria that are located in single longitudinal columns between the myofibrils, with no specific relationship to CRUs (Figure 3; see [34, 35]). Even in the flight muscles of hummingbirds, mitochondria are not especially associated with CRUs. It is likely however that 


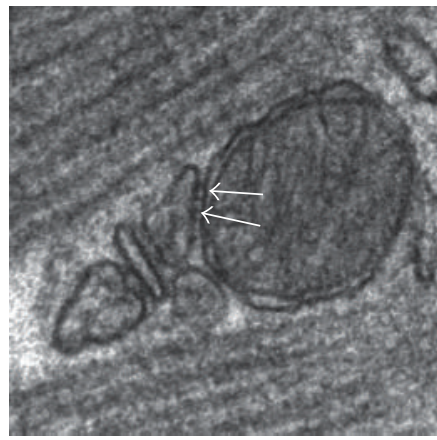

(a)

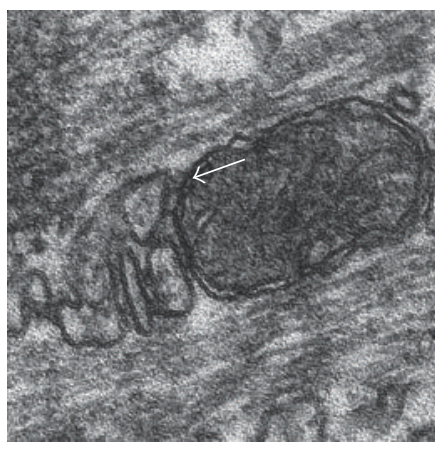

(c)

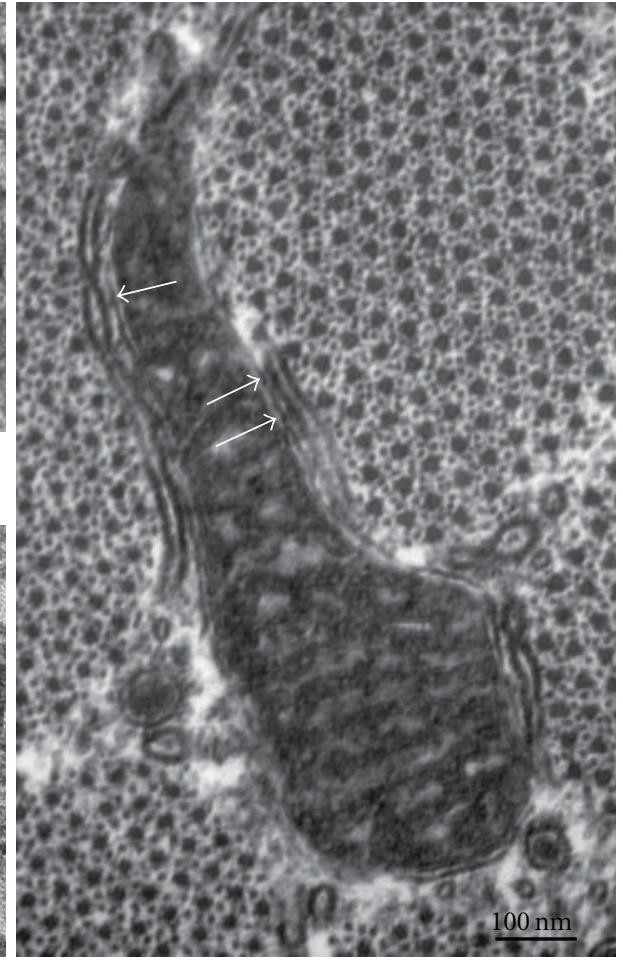

(b)

FIGURE 2: Higher magnifications highlighting association of mitochondria and SR (a)-(c). Tethering connections anchor mitochondria to the jSR of the triads (CRUs) in images of mouse EDL ((a) and (c), white arrows). Similar tethers (white arrows) anchor mitochondria to the longitudinal SR, not necessarily to CRUs, in the leg muscle from a finch (b).

the disposition of longitudinal mitochondria in the intermyofibrillar spaces is not entirely random. Each mitochondrion is in very close proximity to SR elements along its length, and it seems to be anchored to them by tethers that greatly resemble those in mammalian muscles (compare arrows in Figure 2(b) with Figures 2(a) and 2(c)). We argue that tethering to SR is responsible for holding the mitochondria within the intermyofibrillar spaces and keeping them from moving out and aggregating into subplasmalemmal clusters.

Longitudinal mitochondria have a chance of being at a short distance from one or more CRUs along their length as they run past the level of the sarcomere at which CRUs are located (Figure 3(b), arrows). The frequency of such encounters depends on the size of the mitochondrion and on the frequency of triads along the T tubule network. Note also that this configuration offers a very limited close proximity between the mitochondrion surface and the CRU.

Strikingly, the frequency of mitochondria in leg and flight muscles of the finch is actually lower than in the mouse leg muscle, despite the apparent similarity in the fast and continuously active movements in these two species.

In the species mentioned above, there also seems to be no accumulation of mitochondria in proximity of capillaries even within the more aerobic muscles. Such accumulations however are present in highly specialized muscles. The mitochondria respiration rates in the flight muscle of hummingbirds is about two times higher than that of locomotory mus- cles in mammals running at their maximum aerobic capacities. Capillary volume density is correspondingly higher, and mitochondria are packed so highly that they almost compromise the ability to produce force by crowding out myofibrils [36]. Most relevant is the fact that mitochondria are precisely clustered at a high density right at the capillary borders [37].

3.3. Reptiles. We examined the body muscles of two snakes (Boa constrictor and Nerodia sipedon) and the leg muscles of a lizard (Anolis carolinensis) as examples of reptile muscles that are used for a variety of slow to fast movements. The slower tortoise muscles have also been described [38]. Three different types of muscle fibres in Boa c. showed the distinctive characteristics of tonic (large myofibrils, limited membrane systems, and few mitochondria) probably used when the snake crushes its prey, phasic "red" (smaller myofibrils, slightly higher mitochondria content) probably used when the snake moves around for longer periods of time, and phasic "white" (smaller myofibrils, low mitochondria content) probably used when the snake strikes a prey. The lizard leg muscles had small myofibrils and frequent triads as to be expected from the very fast movements of these graceful animals when in search of pray.

A noticeable feature of these reptilian muscles is a scarcity of mitochondria in keeping with the general motile pattern of prolonged immobility alternated with brief periods of fast activity. The overall distribution pattern is the same as 


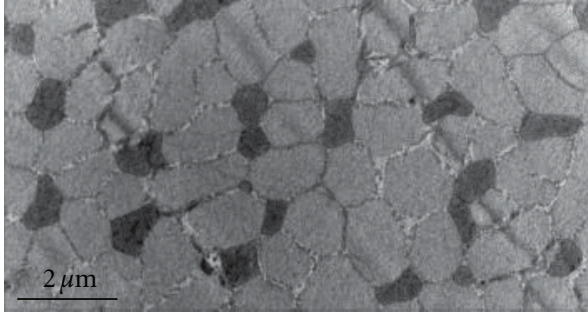

(a)

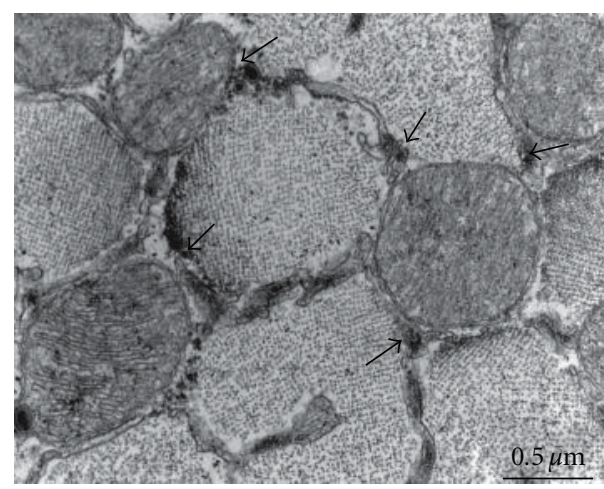

(b)

FIGURE 3: Breast muscle from the finch shown in cross-sections (a) and (b). In the finch, as in other birds, mitochondria are located in single longitudinally oriented rows between the myofibrils. A limited proximity between mitochondria and CRUs (arrows in (b)) occurs where the mitochondria cross the level at which $\mathrm{T}$ tubules are located. Accumulations of mitochondria at the fiber edges are not present in the flight muscles of many birds, indicating that the mitochondria are not free to escape from the myofibrillar domains of the fibers, probably due to tethering; see Figure 2.

in birds: and the same comments apply: mitochondria are located in single columns occupying the intermyofibrillar spaces, with no special relationship to CRUs and no accumulation at capillaries (Figure 4, from the lizard).

As in the case of birds, a fast, continuously active muscle provides an ultimate example of functionally designed organization. "Sound production is one of the most energetically costly activities in animals" [39]. Despite extremely reduced tension production that minimizes contractile use of ATP [39] and some other mechanical tradeoffs strategies [40], the rattlesnakes tail shaker muscle is a fascinating example of the adaptations necessary to produce and maintain extremely fast contraction-relaxation rates [41]. Mitochondria are indeed at high density in these muscles and large clusters are for the most part fairly close to capillaries, but they do not bear a specific spatial relationship to CRUs [42].

3.4. Amphibia. In muscles of amphibia, as exemplified by various species of Rana ( $R$. pipiens, $R$ temporaria $[43,44]$; see also [45] for Rana japonica and [46] for Rana nigromaculata), the large majority of the mitochondria tend to be located between the myofibrils [46]. Frog mitochondria are fairly large; they are positioned in single file between the myofibrils and an individual organelle may run for the length of one-

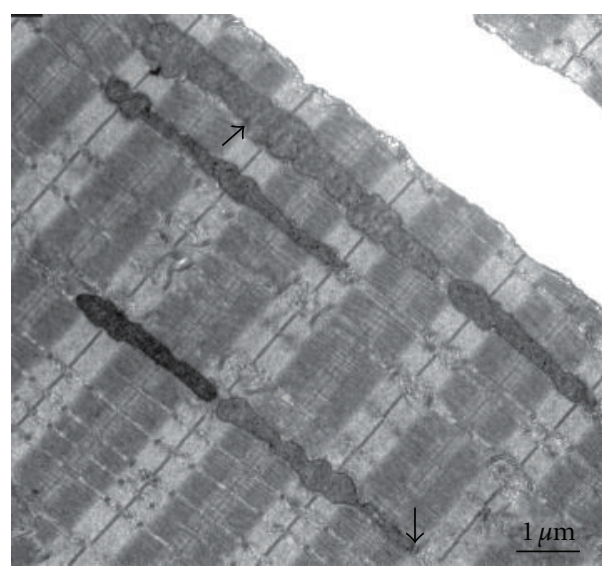

(a)

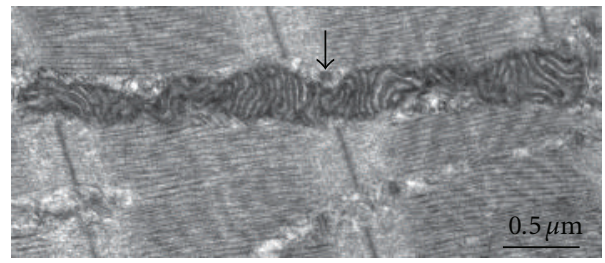

(b)

FIgURE 4: Longitudinal sections of leg muscles from a small lizard, as an example from reptiles. The myofibrils are small, and the CRUs (triads) are frequent, as expected from a rapid muscle. Mitochondria are not frequent, and they are located in single longitudinal rows between the myofibrils. An occasional proximity to CRU occurs when the mitochondria cross the T tubule network (arrows, see also Figures 3 and 5 for birds and amphibia).

two sarcomeres (Figure 5). There is no peripheral clustering at the fiber edge. The disposition is the same in sartorius and gastrocnemius where mitochondria are unusually scarce (Figure 5) and in the iliofibularis where they are more frequent.

In general, none of the Rana muscles have a content of mitochondria comparable to that of rat or mouse, indicating a limited reliance on oxidative phosphorylation and there are no descriptions of muscle with an extensive mitochondria accumulation. Tonic fibers that sustain prolonged periods of activity (e.g., those used in the mating amplexus) have an even lower density of mitochondria, in keeping with the fact that although the contractions are prolonged, they involve very slow cycling cross-bridges and thus require a limited amount of ATP.

3.5. Fish. At the lower end of the vertebrate subphylum, we collected images from tail musculature of a variety of fish, from primitive to more advanced. For example, see [47-50]. All muscles fibers, including the "red" ones used for powering swimming motion [51], contain elongated mitochondria that are either located in longitudinal slits between myofibrils (Figure 6(a)) or, more frequently, at the fibers' edges, under the plasmalemma (Figure 6(b)). An interesting variation relative to birds, reptiles, and amphibia is the fact that intermyofibrillar mitochondria are most 


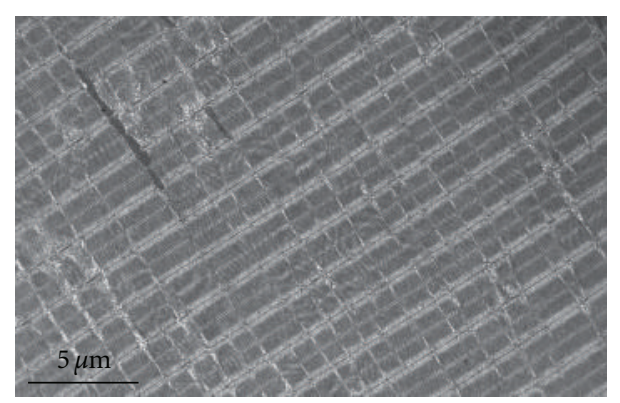

(a)

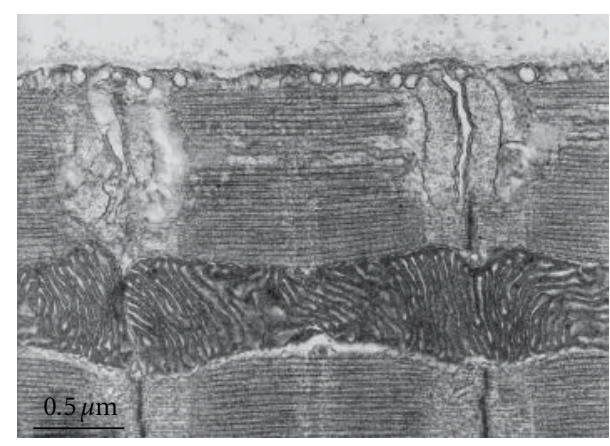

(b)

Figure 5: Images from the frog gastrocnemius (a) and sartorius (b) in longitudinal sections. The predominant location of mitochondria is similar to that of birds and reptiles, again providing limited contacts with CRUs. Note overall scarcity of mitochondria in these muscles gastrocnemius. Others have more frequent mitochondria but still no large clusters at the periphery.

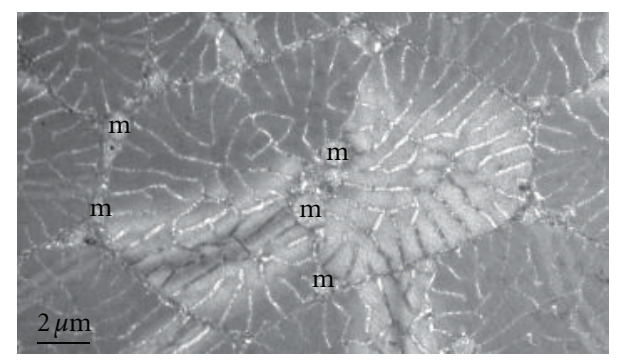

(a)

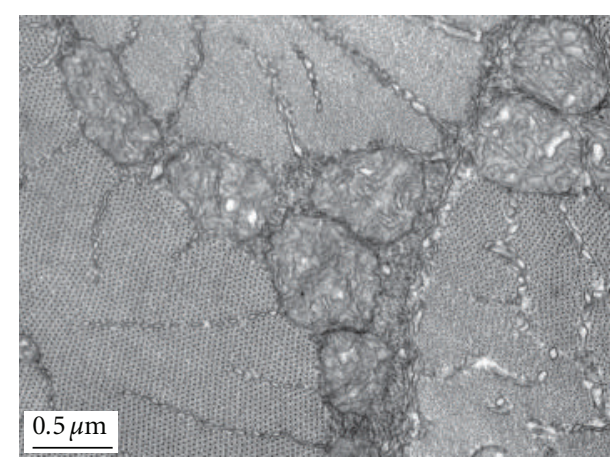

(b)

Figure 6: Cross-sections of small fibers in zebrafish. These images illustrate two characteristics of mitochondria $(\mathrm{m})$ disposition in fish muscles. There is a tendency for clustering of the organelles in small groups that are mostly segregated to the fibers' edges. In this manner, proximity of the mitochondrion' surface to CRUs is quite limited. often, particularly if more numerous, collected into small, longitudinally aligned clusters. This tendency is strongly emphasized in the case of cold adaptation in fish such as the striped bass, that results in an increase of mitochondrial volume density by as much as $230 \%$ [52]. While in the fish exposed to warmer water the mitochondria are positioned in single longitudinal rows between the myofibrils, in the cold-adapted fish the mitochondria are accumulated in large clusters both between the myofibrils and at the fiber periphery. The direct effect of clustering is that of decreasing the probability that an individual mitochondrion comes to close proximity to a CRU. Higher oxidative capacity relates to higher mitochondrial content, but not to a better contact with CRUs $[53,54]$.

Interestingly, in superfast sound-producing swimbladder musculature of Opsanus tau (toadfish), where the mitochondria are not frequent $[55,56]$ and of Porichthys notatus (midshipman) where they are quite abundant [57], mitochondria are almost completely excluded from the regions of the fiber containing myofibrils and placed instead in the central core and outer subplasmalemmal ring. The physiology of these fast-acting muscle fibers, like those of the rattlesnake, has been modified for superfast repetitive contractions with little force production [58], thus reducing their ATP requirements.

\section{Conclusions}

This brief comparative look at skeletal muscles shows that a well-regulated, close, extensive association between mitochondria and CRUs is present only in mammalian skeletal muscles. In other vertebrate muscles, the mitochondriaCRU proximity is far less extensive and less well regulated. Since the presumably privileged mitochondrial disposition of mammalian muscles is a relatively late event in evolutionary times, it must confer some advantage, but it may not be essential to muscle function. Apparently, coupling of muscle activation to stimulation of mitochondrial aerobic ATP production must occur even in the absence of the mammalian-type associations.

It is hard to actually pinpoint the precise advantage that extensive mitochondria-CRU association confers. Even assuming that some $\mathrm{Ca}^{2+}$ uptake by mitochondria is absolutely essential, it must be remembered that this uptake must be limited in order to avoid the total collapse of the mitochondrion's inner membrane potential. So, it is not entirely clear that the extensive ER/SR-mitochondria contacts that are present in liver and in mammalian skeletal muscle are necessary and/or advantageous relative to the more fleeting contacts between CRU and mitochondria of muscles in lower vertebrates.

Perhaps the answer, in the case of mammalian skeletal muscle, lies in the fact that mitochondria-CRU contacts foster the differentiation of complex mitochondrial shapes. Increases in cytosolic calcium levels above resting seem to reduce mitochondrial motility [59], so an initial association of large mitochondria with CRUs may have a positive feedback effect, enhancing and stabilizing further associations. Mitochondria that are not tethered to SR and thus end up in peripheral clusters are smaller and not as extensive as the 
CRU-associated ones. During postnatal muscle fiber maturation in mouse, extension of mitochondria into branched pattern and their association with CRUs occur in parallel [22]. A direct effect of this unique mitochondria tendency towards extension for long distances both longitudinally and transversely is the effective spread of information over relatively large regions of the fiber, and this may have some specific advantages. For example, mitochondria-based superoxide flashes (mSOF) in resting and activated mouse FDB fibers occur simultaneously over extended regions reflecting the complex mitochondrial network [60]. Considering that a moderate level of superoxide production has physiological effects (discussed in [60]), coordination of its production is certainly of advantage.

It is, however, also noteworthy that extensive CRUmitochondria contacts are avoided in a mammalian fastacting muscle capable of prolonged activity: the bat cricothyroid, used for echolocation [33]. Additionally, mitochondria tethering is "loosened" in a mouse muscle with a leaky mutation of the RyR [61] emphasizing the inherent risks of a close proximity of mitochondria to $\mathrm{Ca}^{2+}$ sources. Perhaps fish muscles have the best solution. Mitochondria clustering in those muscles has the effect of reducing the portion of each organelle's outline that faces directly towards a $\mathrm{CRU}$, thus reducing the influence of $\mathrm{CRU}^{\prime} \mathrm{Ca}^{2+}$ release on mitochondria and allowing the development of some of the fastest known muscle fibers, where mitochondria interfere very little with $\mathrm{Ca}^{2+}$ cycling.

\section{Abbreviations}

$\begin{array}{ll}\text { CRU: } & \mathrm{Ca}^{2+} \text { release unit } \\ \text { EDL: } & \text { Extensor digitorum longus } \\ \text { FDB: } & \text { Flexor digitorum brevis muscle } \\ \text { EM: } & \text { Electron microscopy } \\ \text { SR: } & \text { Sarcoplasmic reticulum } \\ \text { jSR: } & \text { Junctional SR } \\ \text { T tubule: } & \text { Transverse tubule. }\end{array}$

\section{Acknowledgments}

The authors thank Dr. Angela Dulhunty from the Australian National University for kindly providing a sample of Kangaroo muscle, Mr. Mathew Close for lizard and snake muscles, and Mrs. Inna Martinyuk for electron microscopy support. This work was supported by NIH Grant RO1 H 48093 to C. Franzini Armstrong.

\section{References}

[1] C. S. Rossi and A. L. Lehninger, "Stoichiometry of respiratory stimulation, accumulation oF $\mathrm{Ca}^{++}$and phosphate, and oxidative phosphorylation in rat liver mitochondria," The Journal of Biological hemistry, vol. 239, pp. 3971-3980, 1964.

[2] R. S. Balaban, "Cardiac energy metabolism homeostasis: role of cytosolic calcium," Journal of Molecular and Cellular Cardiology, vol. 34, no. 10, pp. 1259-1271, 2002.

[3] P. S. Brookes, Y. Yoon, J. L. Robotham, M. W. Anders, and S. S. Sheu, "Calcium, ATP, and ROS: a mitochondrial love-hate triangle," American Journal of Physiology-Cell Physiology, vol. 287, no. 4, pp. C817-C833, 2004.

[4] R. Rizzuto, A. W. M. Simpson, M. Brini, and T. Pozzan, "Rapid changes of mitochondrial $\mathrm{Ca}^{2+}$ revealed by specifically targeted recombinant aequorin," Nature, vol. 358, no. 6384, pp. 325-327, 1992.

[5] G. Hajnoczky, L. D. Robb-Gaspers, M. B. Seitz, and A. P. Thomas, "Decoding of cytosolic calcium oscillations in the mitochondria," Cell, vol. 82, no. 3, pp. 415-424, 1995.

[6] B. Chance, G. R. Williams, W. F. Holmes, and J. Higgins, "Respiratory enzymes in oxidative phosphorylation. V. A mechanism for oxidative phosphorylation," The Journal of Biological Chemistry, vol. 217, no. 1, pp. 439-451, 1955.

[7] E. Carafoli, C. S. Rossi, and A. L. Lehninger, "Uptake of Adenine Nucleotides by Respiring Mitochondria During Active Accumulation of $\mathrm{Ca}^{++}$and Phosphate," The Journal of Biological Chemistry, vol. 240, pp. 2254-2261, 1965.

[8] A. Scarpa and P. Graziotti, "Mechanisms for intracellular calcium regulation in heart. I. Stopped flow measurements of $\mathrm{Ca}^{++}$uptake by cardiac mitochondria," Journal of General Physiology, vol. 62, no. 6, pp. 756-772, 1973.

[9] R. Rizzuto, M. Brini, M. Murgia, and T. Pozzan, "Microdomains with high $\mathrm{Ca}^{2+}$ close to IP3-sensitive channels that are sensed by neighboring mitochondria," Science, vol. 262, no. 5134, pp. 744-747, 1993.

[10] R. Rizzuto, P. Pinton, W. Carrington et al., "Close contacts with the endoplasmic reticulum as determinants of mitochondrial $\mathrm{Ca}^{2+}$ responses," Science, vol. 280, no. 5370, pp. 17631766, 1998.

[11] R. Rizzuto and T. Pozzan, "Microdomains of intracellular $\mathrm{Ca} 2+$ : molecular determinants and functional consequences," Physiological Reviews, vol. 86, no. 1, pp. 369-408, 2006.

[12] G. Csordás, P. Várnai, T. Golenár et al., "Imaging interorganelle contacts and local calcium dynamics at the ERmitochondrial interface," Molecular Cell, vol. 39, no. 1, pp. 121-132, 2010.

[13] C. A. Mannella, K. Buttle, B. K. Rath, and M. Marko, "Electron microscopic tomography of rat-liver mitochondria and their interactions with the endoplasmic reticulum," BioFactors, vol. 8, no. 3-4, pp. 225-228, 1998.

[14] G. C. Shore and J. R. Tata, "Two fractions of rough endoplasmic reticulum from rat liver. I. Recovery of rapidly sedimenting endoplasmic reticulum in association with mitochondria," Journal of Cell Biology, vol. 72, no. 3, pp. 714-725, 1977.

[15] C. García-Pérez, G. Hajnóczky, and G. Csordás, "Physical coupling supports the local $\mathrm{Ca}^{2+}$ transfer between sarcoplasmic reticulum subdomains and the mitochondria in heart muscle," Journal of Biological Chemistry, vol. 283, no. 47, pp. 3277132780, 2008.

[16] G. Szabadkai, K. Bianchi, P. Várnai et al., "Chaperonemediated coupling of endoplasmic reticulum and mitochondrial Ca ${ }^{2+}$ channels," Journal of Cell Biology, vol. 175, no. 6, pp. 901-911, 2006.

[17] R. Rudolf, M. Mongillo, P. J. Magalhäes, and T. Pozzan, "In vivo monitoring of $\mathrm{Ca}^{2+}$ uptake into mitochondria of mouse skeletal muscle during contraction," Journal of Cell Biology, vol. 166, no. 4, pp. 527-536, 2004.

[18] V. K. Sharma, V. Ramesh, C. Franzini-Armstrong, and S. S. Sheu, "Transport of $\mathrm{Ca}^{2+}$ from sarcoplasmic reticulum to mitochondria in rat ventricular myocytes," Journal of Bioenergetics and Biomembranes, vol. 32, no. 1, pp. 97-104, 2000.

[19] J. M. Gillis, "Inhibition of mitochondrial calcium uptake slows down relaxation in mitochondria-rich skeletal muscles," 
Journal of Muscle Research and Cell Motility, vol. 18, no. 4, pp. 473-483, 1997.

[20] E. V. Isaeva and N. Shirokova, "Metabolic regulation of $\mathrm{Ca}^{2+}$ release in permeabilized mammalian skeletal muscle fibres," Journal of Physiology, vol. 547, part 2, pp. 453-462, 2003.

[21] T. Ogata and Y. Yamasaki, "Ultra-high-resolution scanning electron microscopy of mitochondria and sarcoplasmic reticulum arrangement in human red, white, and intermediate muscle fibers," Anatomical Record, vol. 248, no. 2, pp. 214-223, 1997.

[22] S. Boncompagni, A. E. Rossi, M. Micaroni et al., "Mitochondria are linked to calcium stores in striated muscle by developmentally regulated tethering structures," Molecular Biology of the Cell, vol. 20, no. 3, pp. 1058-1067, 2009.

[23] V. M. Shkryl and N. Shirokova, "Transfer and tunneling of $\mathrm{Ca}^{2+}$ from sarcoplasmic reticulum to mitochondria in skeletal muscle," Journal of Biological Chemistry, vol. 281, no. 3, pp. 1547-1554, 2006.

[24] A. Rambourg and D. Segretain, "Three-dimensional electron microscopy of mitochondria and endoplasmic reticulum in the red muscle fiber of the rat diaphragm," Anatomical Record, vol. 197, no. 1, pp. 33-48, 1980.

[25] T. Ogata and Y. Yamasaki, "Scanning electron-microscopic studies on the three-dimensional structure of mitochondria in the mammalian red, white and intermediate muscle fibers," Cell and Tissue Research, vol. 241, no. 2, pp. 251-256, 1985.

[26] A. E. Rossi, S. Boncompagni, and R. T. Dirksen, "Sarcoplasmic reticulum-mitochondrial symbiosis: bidirectional signaling in skeletal muscle," Exercise and Sport Sciences Reviews, vol. 37, no. 1, pp. 29-35, 2009.

[27] T. M. Williams, G. P. Dobson, O. Mathieu-Costello, D. Morsbach, M. B. Worley, and J. A. Phillips, "Skeletal muscle histology and biochemistry of an elite sprinter, the African cheetah," Journal of Comparative Physiology, B, vol. 167, no. 8, pp. 527-535, 1997.

[28] S. Boncompagni, H. Kern, K. Rossini et al., "Structural differentiation of skeletal muscle fibers in the absence of innervation in humans," Proceedings of the National Academy of Sciences of the United States of America, vol. 104, no. 49, pp. 19339-19344, 2007.

[29] B. R. Eisenberg, A. M. Kuda, and J. B. Peter, "Stereological analysis of mammalian skeletal muscle. I. Soleus muscle of the adult guinea pig," Journal of Cell Biology, vol. 60, no. 3, pp. 732-754, 1974.

[30] A. G. Engel and B. Q. Banker, "Ultrastructural changes in diseased muscle," in Myology, A. G. Engel and C. FranziniArmstrong, Eds., vol. 1, chapter 31, pp. 749-888, McGrawHill, 3rd edition, 2004.

[31] S. Boncompagni, L. d'Amelio, S. Fulle, G. Fanò, and F. Protasi, "Progressive disorganization of the excitation-contraction coupling apparatus in aging human skeletal muscle as revealed by electron microscopy: a possible role in the decline of muscle performance," Journals of Gerontology, Series A, vol. 61, no. 10, pp. 995-1008, 2006.

[32] O. Mathieu-Costello, S. Morales, J. Savolainen, and M. Vornanen, "Fiber capillarization relative to mitochondrial volume in diaphragm of shrew," Journal of Applied Physiology, vol. 93, no. 1, pp. 346-353, 2002.

[33] J. P. Revel, "The sarcoplasmic reticulum of the bat cricothroid muscle," The Journal of cell biology, vol. 12, pp. 571-588, 1962.

[34] A. F. Howatson, "The structure of pigeon breast muscle mitochondria," The Journal of Biophysical and Biochemical Cytology, vol. 2, supplement 4, pp. 363-368, 1956.
[35] S. G. Page, "Structure and some contractile properties of fast and slow muscles of the chicken," Journal of Physiology, vol. 205, no. 1, pp. 131-145, 1969.

[36] I. Grinyer and J. C. George, "Some observations on the ultrastructure of the hummingbird pectoral muscles," Canadian Journal of Zoology, vol. 47, no. 5, pp. 771-773, 1969.

[37] R. K. Suarez, J. R. B. Lighton, G. S. Brown, and O. MathieuCostello, "Mitochondrial respiration in hummingbird flight muscles," Proceedings of the National Academy of Sciences of the United States of America, vol. 88, no. 11, pp. 4870-4873, 1991.

[38] S. G. Page, "Fine structure of tortoise skeletal muscle," Journal of Physiology, vol. 197, no. 3, pp. 709-715, 1968.

[39] K. E. Conley and S. L. Lindstedt, "Minimal cost per twitch in rattlesnake tall muscle," Nature, vol. 383, no. 6595, pp. 71-72, 1996.

[40] B. R. Moon, J. J. Hopp, and K. E. Conley, "Mechanical tradeoffs explain how performance increases without increasing cost in rattlesnake tailshaker muscle," Journal of Experimental Biology, vol. 205, part 5, pp. 667-675, 2002.

[41] L. C. Rome, D. A. Syme, S. Hollingworth, S. L. Lindstedt, and S. M. Baylor, "The whistle and the rattle: the design of sound producing muscles," Proceedings of the National Academy of Sciences of the United States of America, vol. 93, no. 15, pp. 8095-8100, 1996.

[42] P. J. Schaeffer, K. E. Conley, and S. L. Lindstedt, "Structural correlates of speed and endurance in skeletal muscle: the rattlesnake tailshaker muscle," Journal of Experimental Biology, vol. 199, part 2, pp. 351-358, 1996.

[43] C. Franzini-Armstrong, "Studies of the triad. 3. Structure of the junction in fast twitch fibers," Tissue and Cell, vol. 47, no. 2, pp. 488-499, 1970.

[44] C. Franzini-Armstrong, "Studies of the triad. IV. Structure of the junction in frog slow fibers," Journal of Cell Biology, vol. 56, no. 1, pp. 120-128, 1973.

[45] I. Sato, K. Konishi, M. Sunohara, and A. Mikami, "Enzyme activities and morphology of Japanese brown frog (Rana japonica) mitochondria in the tibialis anterior muscle during hibernation and active life," Canadian Journal of Zoology, vol. 79, no. 7, pp. 1316-1321, 2001.

[46] T. Ogata and Y. Yamasaki, "High-resolution scanning electronmicroscopic studies on the three-dimensional structure of mitochondria and sarcoplasmic reticulum in the different twitch muscle fibers of the frog," Cell and Tissue Research, vol. 250, no. 3, pp. 489-497, 1987.

[47] C. Franzini-Armstrong and K. R. Porter, "Sarcolemmal invaginations constituting the T system in fish muscle fibers," Journal of Cell Biology, vol. 22, pp. 675-696, 1964.

[48] C. Franzini-Armstrong, W. F. Gilly, E. Aladjem, and D. Appelt, "Golgi stain identifies three types of fibres in fish muscle," Journal of Muscle Research and Cell Motility, vol. 8, no. 5, pp. 418-427, 1987.

[49] V. Di Biase and C. Franzini-Armstrong, "Evolution of skeletal type e-c coupling: a novel means of controlling calcium delivery," Journal of Cell Biology, vol. 171, no. 4, pp. 695-704, 2005.

[50] J. Schredelseker, V. Di Biase, G. J. Obermair et al., "The $\beta 1$ a subunit is essential for the assembly of dihydropyridinereceptor arrays in skeletal muscle," Proceedings of the National Academy of Sciences of the United States of America, vol. 102, no. 47, pp. 17219-17224, 2005.

[51] M. A. Freadman, "Role partitioning of swimming musculature of striped Bass Morone saxatilis Walbaum and Bluefish, Pomatomus saltatrix L.," Journal of Fish Biology, vol. 15, no. 4, pp. 417-423, 1979. 
[52] S. Egginton and B. D. Sidell, "Thermal acclimation induces adaptive changes in subcellular structure of fish skeletal muscle," American Journal of Physiology, vol. 256, no. 1, part 2, pp. R1-R9, 1989.

[53] K. M. O'Brien, C. Skilbeck, B. D. Sidell, and S. Egginton, "Muscle fine structure may maintain the function of oxidative fibres in haemoglobinless Antarctic fishes," Journal of Experimental Biology, vol. 206, part 2, pp. 411-421, 2003.

[54] I. A. Johnston, J. Calvo, H. Guderley, D. Fernandez, and L. Palmer, "Latitudinal variation in the abundance and oxidative capacities of muscle mitochondria in perciform fishes," Journal of Experimental Biology, vol. 201, part 1, pp. 1-12, 1998.

[55] K. E. Loesser, J. Rafi, and M. L. Fine, "Embryonic, juvenile, and adult development of the toadfish sonic muscle," Anatomical Record, vol. 249, no. 4, pp. 469-477, 1997.

[56] D. Appelt, V. Shen, and C. Franzini-Armstrong, "Quantitation of Ca ATPase, feet and mitochondria in superfast muscle fibres from the toadfish, Opsanus tau," Journal of Muscle Research and Cell Motility, vol. 12, no. 6, pp. 543-552, 1991.

[57] M. K. Lewis, P. C. Nahirney, V. Chen et al., "Concentric intermediate filament lattice links to specialized Z-band junctional complexes in sonic muscle fibers of the type I male midshipman fish," Journal of Structural Biology, vol. 143, no. 1, pp. 56-71, 2003.

[58] L. C. Rome and S. L. Lindstedt, "The quest for speed: muscles built for high-frequency contractions," News in Physiological Sciences, vol. 13, no. 6, pp. 261-268, 1998.

[59] M. Yi, D. Weaver, and G. Hajnóczky, "Control of mitochondrial motility and distribution by the calcium signal: a homeostatic circuit," Journal of Cell Biology, vol. 167, no. 4, pp. 661-672, 2004.

[60] L. Wei, G. Salahura, S. Boncompagni et al., "Mitochondrial superoxide flashes: metabolic biomarkers of skeletal muscle activity and disease," FASEB Journal, vol. 25, pp. 3068-3078, 2011.

[61] S. Boncompagni, A. E. Rossi, M. Micaroni et al., "Characterization and temporal development of cores in a mouse model of malignant hyperthermia," Proceedings of the National Academy of Sciences of the United States of America, vol. 106, no. 51, pp. 21996-22001, 2009. 

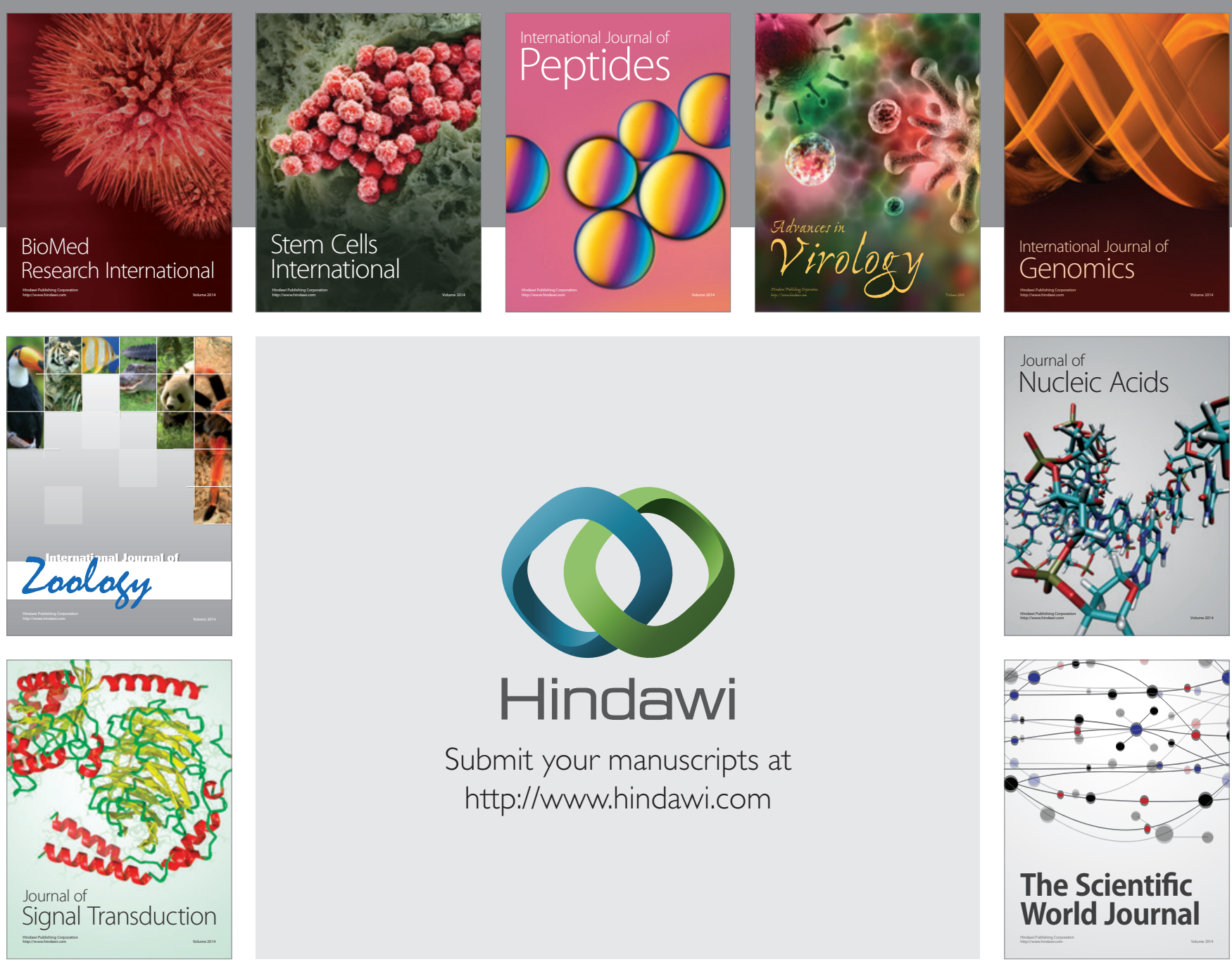

Submit your manuscripts at

http://www.hindawi.com
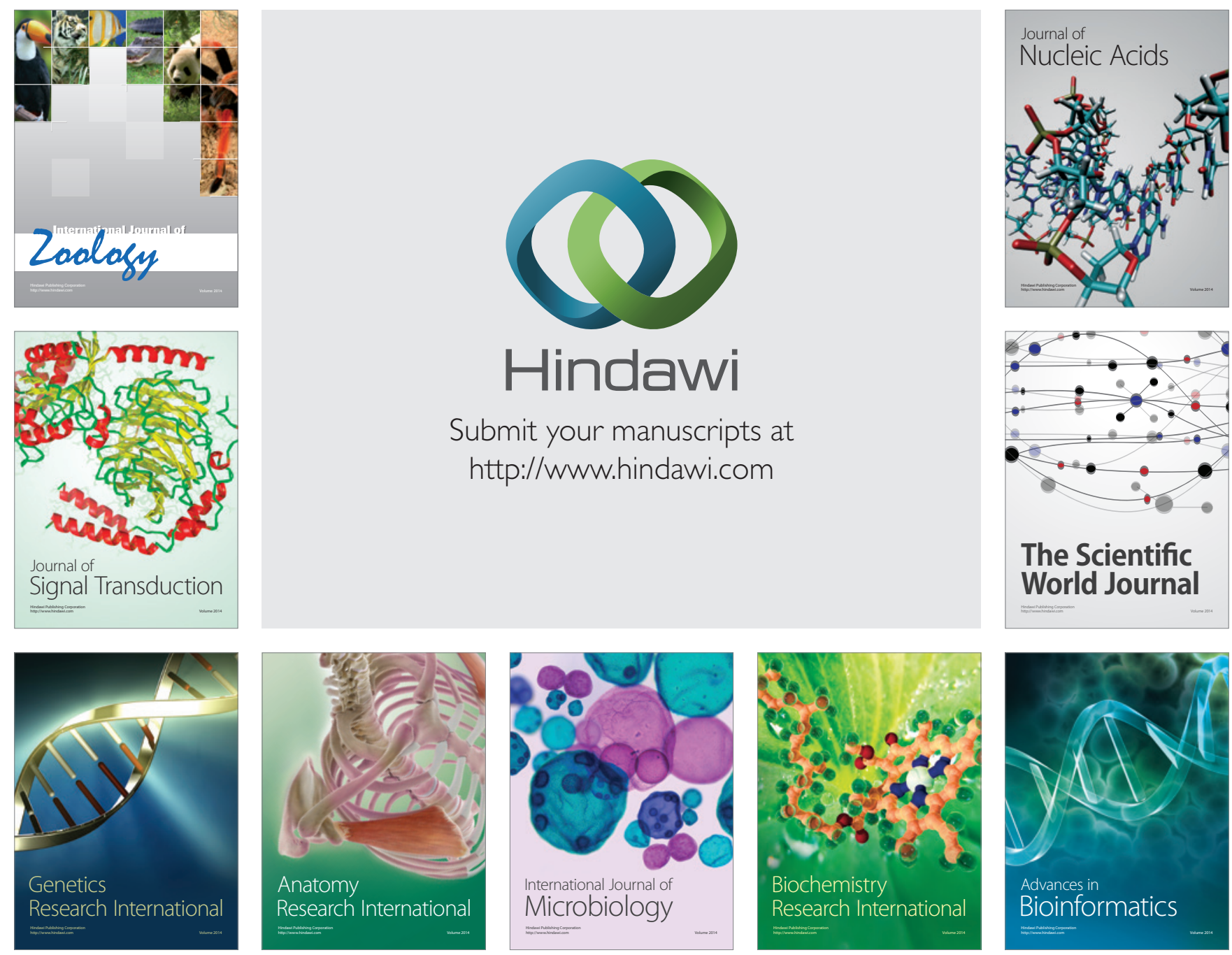

The Scientific World Journal
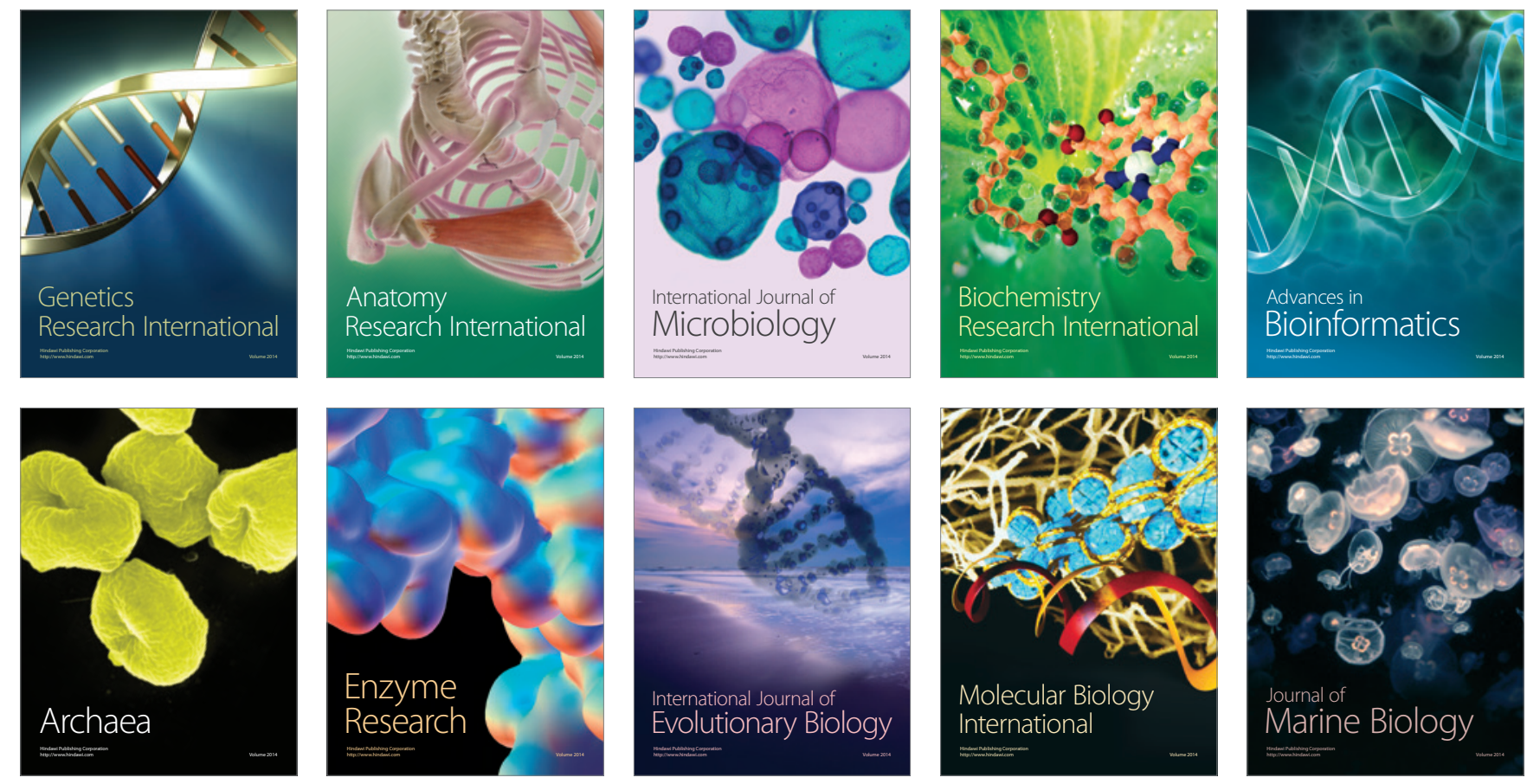